TRABAJOS ORIGINALES

\title{
Crecimiento por ramificación basal en dos especies de palmeras huicungo, Astrocaryum carnosum y A. huicungo
}

\section{Growth by basal branching in two species of Huicungo palms, Astrocaryum carnosum and A. huicungo}

\section{Miguel Machahua, Francis Kahn y Betty Millán}

Museo de Historia Natural, Universidad Nacional Mayor de San Marcos, Av. Arenales 1256, Jesús María, Lima 11, Perú.

Email Miguel Machahua: m.machahua@gmail.com

Email Francis Kahn: franciskahn@orange.fr

Email Betty Millán: bmillans@gmail.com

\begin{abstract}
Resumen
El proceso de ramificación basal de Astrocaryum carnosum y A. huicungo es descrito y su frecuencia y expresión estructural es analizado en el nivel poblacional. Dos poblaciones de palmeras fueron utilizadas en este estudio, A. carnosum del valle del Alto Huallaga y A. huicungo del valle del Alto Mayo. Los sistemas de ramificación subterráneos fueron examinados y dibujados. Para ambas especies, se establecieron 25 cuadrantes de $20 x 20$ $\mathrm{m}$, en los cuales se contó el número de individuos solitarios y cespitosos, y para estos últimos se registró el número de ejes que conformaban el individuo. Los ejes tanto de los individuos solitarios como de los cespitosos se repartieron por estadio de desarrollo (plántula/juvenil-1/juvenil-2/adulto). El proceso de ramificación basal comienza en individuos al estadio juvenil-2, no se observó en juvenil-1, ni en plántula. $A$. huicungo presentó mayor densidad de ejes producido por ramificación basal que $A$. carnosum. Este proceso de ramificación en las dos especies por formaciones de clones a partir de rizomas cortos permite una explotación óptima del espacio y asegura la continuidad de la población en el tiempo. Sin embargo su papel en la propagación espacial de la población es muy reducido, siendo los rizomas de muy poca extensión.
\end{abstract}

Palabras clave: Astrocaryum; Arecaceae; palmera cespitosa; Amazonia peruana.

\section{Abstract}

The basal branching process of Astrocaryum carnosum and $A$. huicungo is described and its rate and structural expression at the population level is analyzed. Two populations of palms were used in this study, $A$. carnosum from the Alto Huallaga valley and $A$. huicungo from the Alto Mayo valley. Branching systems underground were exposed and drawn. For both species, it was established 25 quadrants of $20 \times 20 \mathrm{~m}$, in which were counted (i) the number of solitary and caespitose individuals, and (ii) the numbers of axes composing the caespitose individuals. The axes of solitary and caespitose individuals were distributed by growth stages (seedling/juvenile-1/ juvenile-2/adult). Seedling and juvenile-1 are not caespitose, basal branching starts in juvenile-2. A. huicungo presents a higher density of axes produced by basal branching than $A$. carnosum. This branching process in both species by formations of clones from short rhizomes allows an optimized exploitation of space and ensures the continuity of the population over time. However, its role in the spatial propagation of the species is very limited by the shortness of the rhizomes.

Keywords: Astrocaryum; Arecaceae; caespitose habit; Peruvian Amazon.

\section{Citación:}

Machahua M., F. Kahn y B. Millán. 2015. Crecimiento por ramificación basal en dos especies de palmeras huicungo, Astrocaryum carnosum y A. huicungo. Revista peruana de biología 22(1): 071 - 076 (Abril 2015) doi: http://dx.doi.org/10.15381/rpb.v22i1.11123

Presentado: $\quad 16 / 12 / 2014$

Aceptado: $\quad 24 / 02 / 2015$

Publicado online: 24/04/2015
Información sobre los autores:

Los autores declaran que participaron en la elaboración del trabajo realizando: MM: muestreo, análisis de datos y redacción; FK: análisis de datos y redacción; BM: análisis de datos y redacción.

Los autores no incurren en conflictos de intereses.

\section{Fuentes de financiamiento:}

Unión Europea-proyecto FP7 PALMS N²12631; Vicerrectorado de Investigación de la Universidad Nacional Mayor de San Marcos, Proyecto No. 141001061. 


\section{Introducción}

La ramificación basal consiste en la producción de uno o varios ejes vegetativos a partir de yemas laterales ubicadas en la base del primer eje elaborado por la germinación. Es un fenómeno común en palmeras (Holttum 1955, Tomlinson 1973 a, 1973 b, 1990, Hallé et al. 1978). Las especies que desarrollan la ramificación basal se denominan "cespitosas" y aquellas que producen un solo eje durante su vida se denominan "solitarias". La ramificación basal se expresa de diferentes formas según las especies. Algunas son muy ramificadas, mientras otras lo son muy poco. También es posible que no se exprese la ramificación dejando la palmera solitaria a pesar de tener el potencial de ramificación. Resultan varias figuras: (i) palmeras con varios ejes adultos, (ii) palmeras con un solo eje adulto y varios retońos basales, (iii) palmeras con un solo eje adulto sin aparente ramificación pero que reemplazó a un eje anterior desaparecido, y (iv) palmeras con un solo eje sin aparente ramificación en la base pero que mantienen la habilidad para la ramificación basal (Kahn \& de Granville 1992, Balslev et al. 2011).

Astrocaryum carnosum y A. huicungo son dos palmeras conocidas comúnmente en el Perú como "Huicungo" y pertenecen a la sección Huicungo del género Astrocaryum. La sección Huicungo presenta 15 especies, de las cuales 12 especies se encuentran distribuidas en la Amazonía del Perú (Kahn 2008, Kahn et al. 2011). Siete de las 15 especies (47\%) son cespitosas, $A$. carnosum, A. faranae, A. huicungo, A. murumuru, A. scopatum, A. ulei y $A$. urostachys (Kahn \& Millán 2013). El proceso de ramificación basal y su efecto a nivel poblacional en este grupo de palmeras es poco conocido (Kahn y Granvillle 1992, Svenning 2000).

El presente trabajo tiene como objetivos: describir el proceso de ramificación basal, evaluar la frecuencia y expresión estructural de la ramificación basal a nivel poblacional en $A$. carnosum y $A$. huicungo.

\section{Materiales y métodos}

Área de estudio.- El estudio fue realizado en las localidades de Uchiza y Nuevo Progreso en el valle del Alto Huallaga $\left(8^{\circ} 26^{\prime} \mathrm{S}-8^{\circ} 35^{\prime} \mathrm{S}\right.$ y $\left.76^{\circ} 21^{\prime} \mathrm{W}-76^{\circ} 30^{\prime} \mathrm{W}, 350 \mathrm{~m}\right)$ y en la localidad

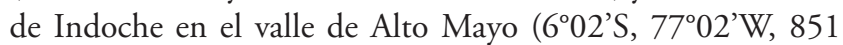
$\mathrm{m}$ ), ambas ubicadas en la Amazonia del Perú (Fig. 1). Las localidades de Uchiza y Nuevo Progreso presentan el clima de un bosque tropical lluvioso con temperatura media anual de $25^{\circ} \mathrm{C}$ y precipitación anual media de $2400 \mathrm{~mm}$; mientras la localidad de Indoche presenta una temperatura media anual de $21{ }^{\circ} \mathrm{C}$ y precipitación anual media de $1400 \mathrm{~mm}$ (BIODAMAZ 2006, PRODATU \& MPT 2008). Las dos áreas de estudio en general presentan una vegetación de bosque tropical intervenido por tala selectiva, intercalado por cultivos y pastizales.

Especies estudiadas.- Astrocaryum carnosum y A. huicungo son palmeras de mediano tamaño que presentan hábito cespitoso (Kahn 2008). Las especies son endémicas del Perú (Millán 2006). Astrocayum carnosum se encuentra en los bosques estacionalmente inundados en el valle del Alto Huallaga entre Tocache y Tingo María; mientras $A$. huicungo se encuentra en las zonas de Rioja-Moyobamba hasta Yurimaguas (Kahn et al. 2011).

Descripción de la ramificación basal.- Para exponer el sistema de ramificación subterráneo de la planta se utilizó el método de Böhm (1979), el cual es uno de los métodos tradicionales para el estudio de raíces de plantas, y consiste en la excavación y

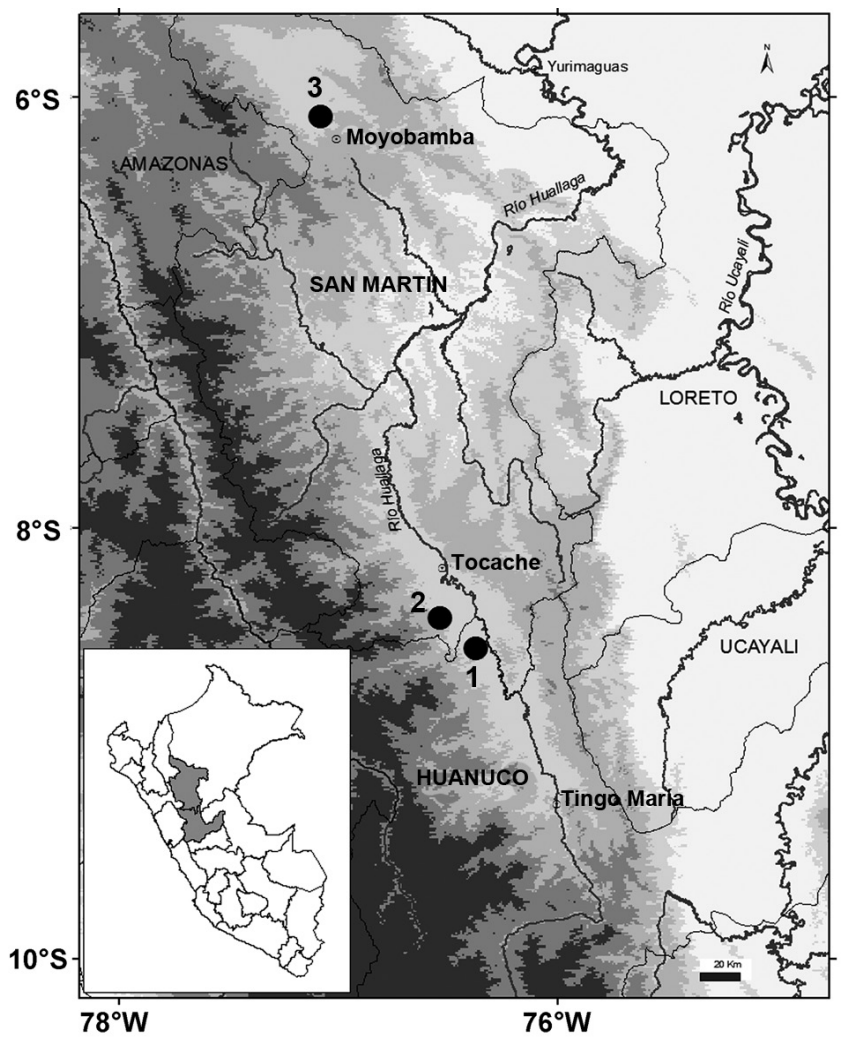

Figura 1. Área de muestreo en el valle del alto Huallaga (1: Nuevo Progreso y 2: Uchiza) y el valle del alto Mayo (3: Indoche) en la Amazonía Peruana.

remoción del suelo circundante al individuo. Se utilizaron herramientas de mano como: palas, picos pequeños, cinceles, tijeras, rompe bloque de tierra y brochas. Se realizaron excavaciones de $0.6 \mathrm{~m}$ de profundidad y $0.8 \mathrm{~m}$ de diámetro para los juveniles; y de $0.9 \mathrm{~m}$ de profundidad y $2 \mathrm{~m}$ de diámetro para los adultos.

El sistema de ramificación de los juveniles fue limpiado y guardado en bolsas para su descripción en gabinete; mientras el sistema de los adultos fue descrito, medido, dibujado y fotografiado en el campo. Por cada especie estudiada, fue observado el sistema de ramificación de cinco individuos en estadio juvenil-2 $\mathrm{y}$ dos individuos en estadio adulto.

Frecuencia y estructura de la ramificación basal a nivel poblacional.- Se establecieron 25 cuadrantes de $20 \times 20$ m por cada especie; los cuadrantes fueron realizados según la metodología propuesta por Anthelme et al. (2010), para lo cual se eligió una palmera adulta como centro del cuadrante y se consideró una distancia mínima de separación entre cada cuadrante de $40 \mathrm{~m}$. En cada cuadrante se contó el número de individuos solitarios o cespitosos por estadio de desarrollo (plántula, juvenil-1, juvenil-2 y adulto). Por cada individuo cespitoso se contó el número de ejes por estadio de desarrollo (tipo plántula, tipo juvenil-1, tipo juvenil-2 y tipo adulto). Para el reconocimiento de los cuatro estadios de desarrollo se adoptó las propuestas de Anthelme et al. (2010): (1) el estadio plántula presenta hojas con lámina foliar entera y bífida, (2) el estadio juvenil-1 presenta hojas de 0,8 a $2 \mathrm{~m}$ de longitud con lámina foliar irregularmente divididas en pinnas, (3) el estadio juvenil-2 presenta hojas de 2 a $4 \mathrm{~m}$ de longitud con lámina foliar regularmente dividida en pinnas y dispuestas en un plano, (4) el estadio adulto presenta hojas de 
más de $4 \mathrm{~m}$ de largo con lámina foliar regularmente dividida en pinnas y dispuestas en un plano.

El análisis de la varianza no paramétrico de Kruskal-Wallis fue usado para evaluar las diferencias observadas entre $A$. carnosum y $A$. huicungo relativas al número de individuos solitarios y cespitosos y al número de ejes por estadio de desarrollo. Los análisis estadísticos fueron realizados con el paquete estadístico InfoStat (Balzarini et al. 2008).

\section{Resultados}

Descripción de la ramificación basal.- La ramificación basal en las dos especies estudiadas se desarrolla a nivel subterráneo. El sistema de ramificación se encuentra unido a través de delgadas conexiones. Cada nuevo eje desarrolla su propio rizoma y sistema de raíces. La forma del rizoma es variable, la más común es la forma de saxofón debido a una fase inicial de crecimiento con geotropismo positivo (Fig. 2).

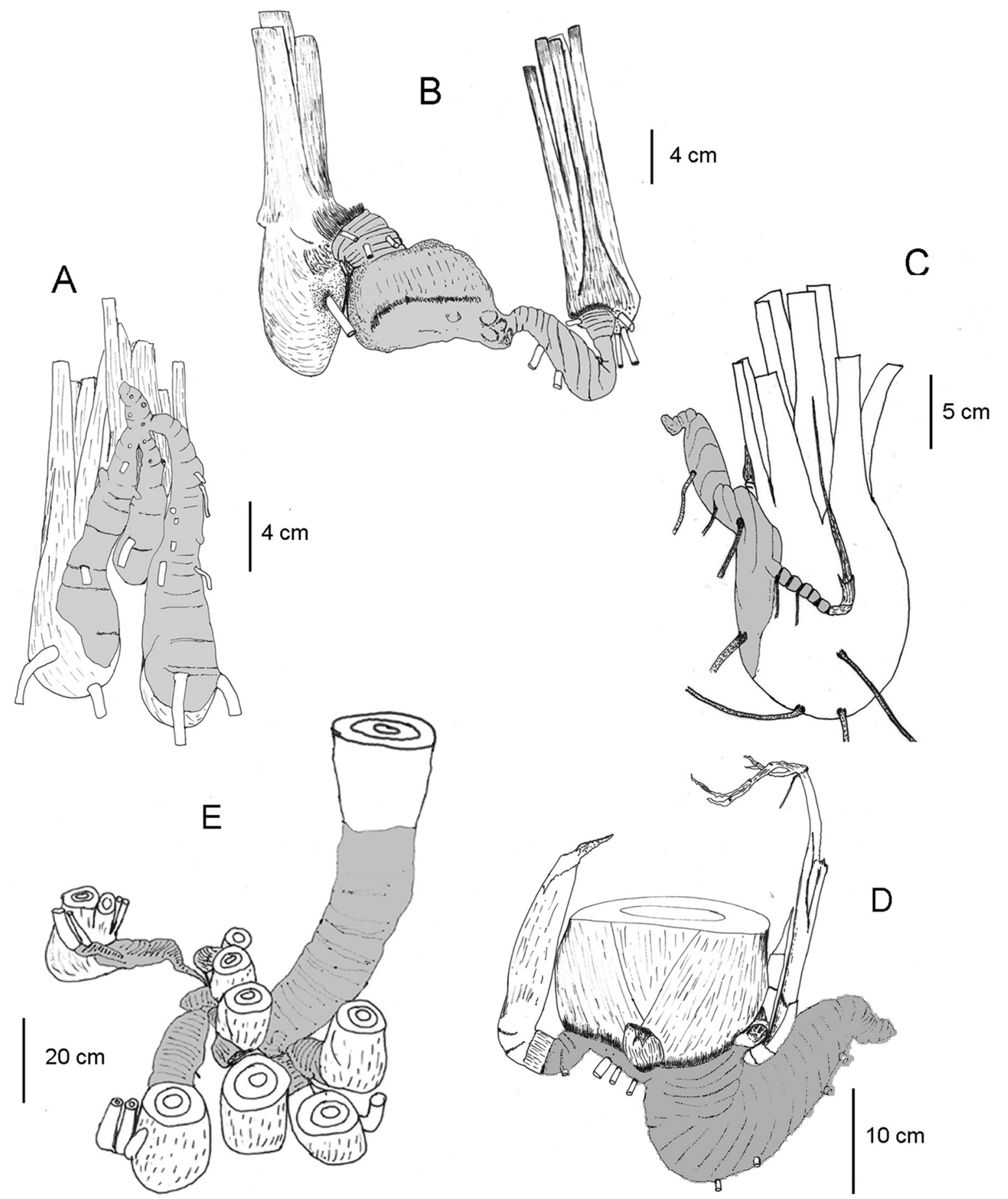

Figura 2. Desarrollo de la ramificación basal. (A): Individuo juvenil-2 de Astrocaryum carnosum con tres ejes tipo juvenil-2. (B): Individuo juvenil-2 de A. huicungo con dos ejes tipo juvenil-2. (C): Individuo juvenil-2 de $A$. carnosum con un eje tipo juvenil-2 y dos brotes. (D): Individuo adulto de $A$. huicungo con un eje tipo adulto y tres brotes. (E): Individuo adulto de $A$. huicungo con 8 ejes tipo adulto y 10 ejes tipo juvenil-1. Las estructuras sombreadas de color gris corresponden al tallo subterráneo y aéreo. 
Tabla 1. Número de individuos solitarios y cespitosos de Astrocaryum carnosum y A. huicungo en 1 ha y 0.04 ha. ( ${ }^{*} p<0.05$, $\left.{ }^{* *} \mathrm{p}<0.01\right)$.

\begin{tabular}{|c|c|c|c|c|c|c|c|}
\hline \multirow[b]{2}{*}{ Especie } & \multicolumn{3}{|c|}{ Solitarios } & \multicolumn{3}{|c|}{ Cespitosos } & \multirow[b]{2}{*}{$p$} \\
\hline & $\begin{array}{l}\text { Estadio de } \\
\text { desarrollo }\end{array}$ & $\begin{array}{c}\text { Número de } \\
\text { individuos } \\
\text { por } 1 \text { ha }\end{array}$ & $\begin{array}{c}\text { Promedio de individuos } \\
\text { por parcela }(0.04 \text { ha), } \\
\text { media } \pm \text { DS(min }- \text { max })\end{array}$ & $\begin{array}{l}\text { Número de } \\
\text { individuos } \\
\text { por } 1 \text { ha }\end{array}$ & $\begin{array}{l}\text { Promedio de individuos } \\
\text { por parcela }(0.04 \text { ha), } \\
\text { media } \pm \text { DS (min - max })\end{array}$ & $\begin{array}{c}\text { Total } \\
\text { individuos por } \\
1 \text { ha }\end{array}$ & \\
\hline \multirow{4}{*}{ A. carnosum } & adulto & 32 & $1.3 \pm 2.2(0-8)$ & 96 & $3.8 \pm 2.8(1-11)$ & 128 & ** \\
\hline & juvenil-2 & 38 & $1.5 \pm 1.5(0-5)$ & 9 & $0.4 \pm 0.7(0-3)$ & 47 & ** \\
\hline & juvenil-1 & 205 & $8.2 \pm 9.1(0-35)$ & 0 & 0 & 205 & ** \\
\hline & plántula & 294 & $11.7 \pm 14.9(0-68)$ & 0 & 0 & 294 & ** \\
\hline \multirow{4}{*}{ A. huicungo } & adulto & 42 & $1.7 \pm 1.8(0-6)$ & 186 & $7.4 \pm 4(0-14)$ & 228 & ** \\
\hline & juvenil-2 & 50 & $2 \pm 1.9(0-9)$ & 25 & $1 \pm 0.9(0-3)$ & 75 & * \\
\hline & juvenil-1 & 299 & $11.9 \pm 9.8(0-37)$ & 0 & 0 & 299 & ** \\
\hline & plántula & 748 & $29.9 \pm 28(0-129)$ & 0 & 0 & 748 & ** \\
\hline
\end{tabular}

En el estadio juvenil-2, el sistema de ramificación subterráneo presenta hasta un primer grado de ramificación, generalmente está constituido por un rizoma principal subterráneo en forma de saxofón y pequeños brotes; o por dos o tres rizomas de igual tamaño. En el estadio adulto el sistema de ramificación suele presentar hasta un cuarto grado de ramificación, esta constituido por rizomas de diferentes tamaños y formas; los nuevos rizomas se encuentran muy cercanos entre ellos.

Frecuencia y estructura de la ramificación basal a nivel poblacional (Tabla 1, 2, Fig. 3).- En Astrocaryum carnosum el número de ejes por individuo cespitoso varió de 2 a 14 y se han registrado un mayor número de individuos cespitosos con 2, 3 y 5 ejes (Fig. 3). En una hectárea, la proporción de individuos cespitosos en estadio adulto fue del $75 \%$ y del $19 \%$ en estadio juvenil-2. A nivel de los cuadrantes de 0.04 ha, el número de los individuos solitarios y cespitosos fueron significativamente diferentes a $\mathrm{p}<0.01$ (Tabla 1). Los 96 individuos adultos cespitosos presentaron en total 434 ejes: 139 (32\%) de tipo adulto, 102 (23\%) de tipo juvenil-2, 193 (44\%) de tipo juvenil-1. Un individuo adulto cespitoso estuvo conformado en promedio por 1.4 ejes de tipo adulto, 1 eje de tipo juvenil-2 y 2 ejes de tipo juvenil-1 (Tabla 2).

En Astrocaryum huicungo el número de ejes por individuo fue de 2 a 78 y se han registrado un mayor número de individuos con 4, 3 y 2 ejes (Fig. 3). En una hectárea, la proporción de individuos cespitosos en estadio adulto fue del $82 \%$ y del $33 \%$ en estadio juvenil-2. A nivel de los cuadrantes de 0.04 ha, el número de los individuos solitarios y cespitosos fueron significativamente diferentes a $\mathrm{p}<0.01$ (Tabla 1 ). Los 186 individuos adultos cespitosos de $A$. huicungo presentaron en total 1335 ejes: $580(43 \%)$ de tipo adulto, 408 (31\%) de tipo juvenil-2, 347 (26\%) de tipo juvenil-1. Un individuo adulto cespitoso estuvo conformado en promedio por 3.1 ejes de tipo adulto, 2.2 ejes de tipo juvenil-2 y 1.8 ejes de tipo juvenil-1 (Tabla 2).

No se registraron ejes tipo plántula en el estadio adulto; sin embargo si fueron reportados en individuos juvenil-2 en las dos especies estudiadas. El eje tipo plántula es muy escaso, debido que se observó sólo en dos individuos en todo el área de estudio.

El número de ejes tipo adulto y juvenil-2 que componen a un individuo adulto cespitoso fue significativamente mayor en A. huicungo que en $A$. carnosum (Tabla 2).

\section{Discusión}

El proceso de ramificación basal.- El sistema de ramificación basal en las dos especies está compuesto de un conjunto de rizomas cortos, unidos a través de delgadas conexiones. La formación de clones a partir de rizomas cortos indica una estrategia de crecimiento que permite una explotación óptima del espacio y asegura la perpetuación de la población en el tiempo (Mendoza \& Franco 1998, Svenning 2000). Sin embargo, en el caso de muerte del tallo principal o la ruptura de las conexiones por degeneración, se ha observado que los nuevos ejes pueden crecer independientemente como ocurre en las palmeras estoloníferas. El crecimiento vegetativo al no pasar por la fase de producción

Tabla 2. Número de ejes de los 96 individuos adultos cespitosos de Astrocaryum carnosum y 186 individuos de A. huicungo. $\left(-p>0.05,{ }^{* *} p<0.01\right)$.

\begin{tabular}{|c|c|c|c|c|c|}
\hline \multirow[b]{2}{*}{ Estadio de desarrollo } & \multicolumn{2}{|c|}{ Astrocaryum carnosum } & \multicolumn{2}{|c|}{ Astrocaryum huicungo } & \multirow[b]{2}{*}{$\mathrm{p}$} \\
\hline & Número de ejes & $\begin{array}{l}\text { Promedio de ejes por } \\
\text { individuo cespitoso. } \\
\text { Media } \pm D S \text { (min - max) }\end{array}$ & Número de ejes & $\begin{array}{l}\text { Promedio de ejes por } \\
\text { individuo cespitoso. } \\
\text { Media } \pm D S \text { (min - max) }\end{array}$ & \\
\hline adulto & 139 & $1.4 \pm 0.6(1-7)$ & 589 & $3.1 \pm 3.9(1-45)$ & ** \\
\hline juvenil-2 & 102 & $1 \pm 1.5(0-7)$ & 408 & $2.2 \pm 2.4(0-21)$ & $* *$ \\
\hline juvenil-1 & 193 & $2 \pm 1.9(0-10)$ & 347 & $1.8 \pm 2(0-10)$ & - \\
\hline plántula & 0 & 0 & 0 & 0 & - \\
\hline Total & 434 & - & 1344 & - & \\
\hline
\end{tabular}




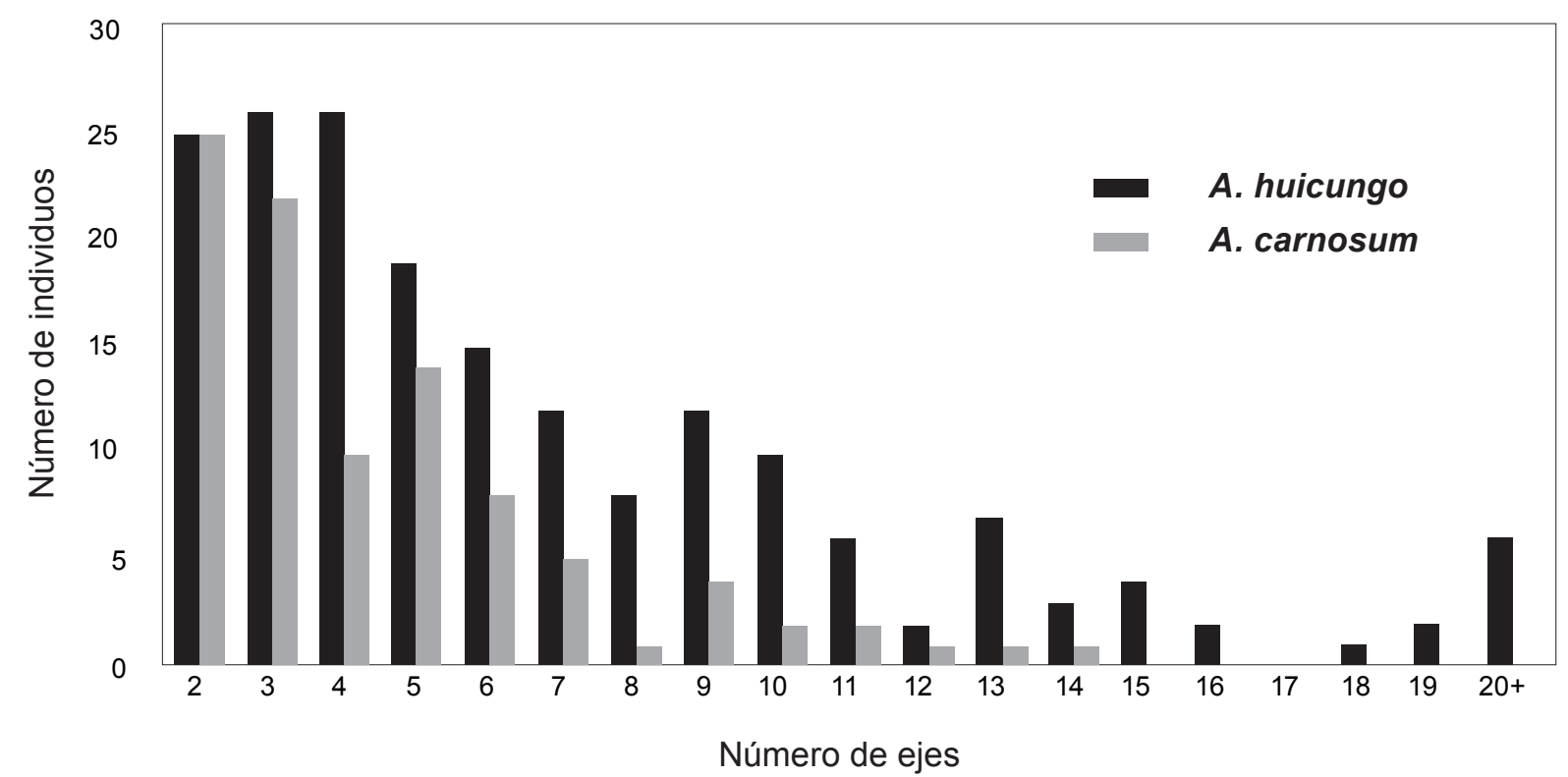

Figura 3. Frecuencia del número de individuos cespitosos adultos por número de ejes en Astrocaryum carnosum y A. huicungo.

de flores evita varios obstáculos, como la no-polinización de las flores pistiladas, el parasitismo muy frecuente de las flores pistiladas de Astrocaryum por larvas de coleópteros Curculionidae que crecen destruyendo el gineceo (Couturier \& Kahn 1992), o el parasitismo también frecuente en las semillas de las palmeras por coleópteros Bruchidae (Delobel et al. 1995)

El proceso de ramificación basal es un evento tardío en el desarrollo de la planta, los primeros estadios corresponden a etapas de establecimiento donde la planta requiere invertir más energía en la formación de rizoma y raíces que en la formación de ejes (De Steven 1989, Tomlinson 1990). En el caso de $A$. carnosum y de $A$. huicungo la producción de nuevos tallos o ejes se inicia comúnmente a partir del estadio juvenil-2 y se intensifica en el estadio adulto. Nuestros resultados difieren de otras especies de palmeras Neotropicales donde se ha encontrado que todo el proceso de ramificación se inicia e intensifica en la fase de establecimiento que corresponde al estadio juvenil (De Steven 1989, Avalos \& Scheider 2011).

La ramificación basal permite aumentar la densidad de ejes adultos en la población como lo muestra una comparación entre especies cespitosas y solitarias. En una hectárea, se registraron 171 y 622 ejes adultos respectivamente en $A$. carnosum y $A$. huicungo, ambas cespitosas (presente estudio), y 67 y 86 ejes adultos respectivamente en $A$. chonta y $A$. javarense, ambas solitarias (Machahua et al. 2014). La mayor densidad de ejes adultos en especies cespitosas soporta la hipótesis de que la ramificación basal es un proceso de crecimiento que prioriza la supervivencia de los individuos y el valor reproductivo para la producción de semillas (Svenning 2000).

Frecuencia y estructura de la ramificación basal.- El porcentaje de individuos adultos cespitosos en $A$. carnosum es $75 \%$ y en $A$. huicungo es $85 \%$, lo cual indica un porcentaje alto en comparación con otras especies del género Astrocaryum. Kahn y Granville (1992) encontraron que el 43\% de los individuos eran cespitosos en $A$. carnosum y el $38 \%$ en $A$. gynacanthum; y Svenning (2000) encontró que el 62\% de los individuos fueron cespitosos en $A$. urostachys en Yasuní, Ecuador. La alta frecuencia de individuos adultos cespitosos promovería el mantenimiento y la expansión de poblaciones locales y la perpetuación de muchos genotipos exitosos (Angevine \& Handel 1986); además indicaría un pasado con un establecimiento no renovado en subsecuentes años y una larga persistencia de esta población (De Steven 1989). Los individuos cespitosos con más ejes podrían ser un buen indicador de vigorosidad; ya que tienen acumulado mayor cantidad de recursos para incrementar más ejes y limitar la mortalidad al desarrollar rizomas y sistemas de raíces (Kouassi et al. 2014).

En el presente estudio no fue posible relacionar factores ambientales con la frecuencia de ramificación. Sin embargo, observamos que el porcentaje de los individuos cespitosos de Astrocaryum carnosum en terrenos pantanosos aumenta con respecto a terrenos de tierra firme; de la misma forma el porcentaje de individuos cespitosos de Astrocaryum huicungo es mayor en terrenos planos que en terrenos con pendiente. Un estudio más amplio y detallado permitirá establecer si esas diferencias son significativas.

\section{Literatura citada}

Angevine M.W. \& S.N. Handel. 1986. Invasion of forest floor space, clonal architecture, and population growth in the perennial herb Clintonia borealis. Journal of Ecology 74: 547-560.

Anthelme F., R. Montúfar-Galárraga, J.C. Pintaud. 2010. Caracterización de la resilencia ecológica de poblaciones de palmeras. Ecología en Bolivia 45(3): 23-29.

Avalos G. \& R.A. Scheider. 2011. Quantification of ramet production in the Neotropical palm Euterpe precatoria (Arecacea) in Costa Rica. Ecotropica 17:31-38.

Balslev H., F. Kahn, B. Millán, J-C. Svenning, et al. 2011. Species Diversity and Growth Forms in Tropical American Palm Communities. The Botanical Review 77: 381-425. DOI : 10.1007/s12229-011-9084-x

Balzarini M.G., L. Gonzalez, M. Tablada, et al. 2008. Infostat. Manual del Usuario, Editorial Brujas, Córdoba, Argentina.

BIODAMAZ. 2006 (en línea). Estrategia Regional de la Diversidad Biológica de San Martín. Documento Técnico. Serie BIODAMAZ-IIAP. Iquitos, Perú. 119 pp. Acceso 03/04/2013.

Böhm W. 1979. Methods of Studying Root Systems (Ecological Studies). Springer-Verlag, Berlin, Heidelberg, New York. 180 p. 
Couturier G. \& F. Kahn. 1992. Notes on the insect fauna on two species of Astrocaryum (Palmae, Cocoeae, Bactridinae) in Peruvian Amazonia with emphasis on potential pests of cultivated palms. Bulletin de l'Institut Français d'Études Andines. No Temático: Las palmeras de los bosques tropicales, 21 (2): 715-726.

Delobel A., G. Couturier, F. Kahn, J.A. Nillson. 1995. Trophic relationships between palms and bruchids (Coleoptera: Bruchidae: Pachymerini) in Peruvian Amazonia. Amazoniana, XIII (3-4): 209-219.

De Steven D. 1989. Genet and ramet demography of Oenocarpus mapora ssp. Mapora, a clonal palm of Panamanian Tropical Moist Forest. Journal of Ecology, 77(2) : 579-596.

Holttum R.E. 1955. Growth-habits of monocotyledons-variation on a theme. Phytomorphology 5:399-413.

Hallé F., R.A.A. Oldeman, P.B. Tomlinson. 1978. Tropical Trees and Forests: An Architectural Analysis. Springer, Berlin.

Kahn F. 2008. El género Astrocaryum (Arecaceae). Revista peruana de biología 15(3), 031- 048. DOI: http://dx.doi.org/10.15381/ rpb.v15i3.2851

Kahn F. \& J.-J. de Granville 1992. Palms in forest ecosystems of Amazonia. Springer Verlag, Berlin, Heidelberg, New York, Tokyo. 226 p.

Kahn F. \& B. Millán. 2013. Las palmeras Huicungo - The Huicungo palms. Fondo Editorial UNMSM, Institut de Recherche pour le Développement, Lima, 173 p.

Kahn F., B. Millán, J.C. Pintaud, et al. 2011. Detailed assessment of the distribution of Astrocaryum sect. Huicungo (Arecaceae) in Peru. Revista peruana de biología 18: 279- 282. DOI: http://dx.doi.org/10.15381/rpb.v18i3.438
Kouassi K.I., S. Barot, K-R. Laossi, et al. 2014. Relationships between ramet and genet dynamics in two clonal palms. Forest Ecology and Management 312:101-107. DOI: http://dx.doi. org/10.1016/j.foreco.2013.10.017

Machahua M., F. Kahn, B. Millán. 2014. Variabilidad vegetativa intra e interespecífica de Astrocaryum chonta y A. javarense (Arecaceae) en Jenaro Herrera, Loreto, Revista peruana de biología 21 (2):139-144. DOI: http://dx.doi.org/10.15381/ rpb.v21i2.9816.

Mendoza A. \& M. Franco. 1998. Sexual reproduction and clonal growth in Reinhardtia gracilis (Palmae), an understory Tropical Palm. American Journal of Botany, 85(4):521-527.

Millán B. 2006. Arecaceae Endémicas del Perú. Revista peruana de biología 13(2): 706-707. DOI: http://dx.doi.org/10.15381/ rpb.v13i2.1940

PRODATU \& MPT. 2008. Tocache hacia el desarrollo sostenible, Plan de Ordenamiento Territorial. Proyecto de Desarrollo Alternativo Tocache Uchiza (PRODATU), Municipalidad Provincial de Tocache (MPT). Lima. 164 pp.

Svenning J.C. 2000. Growth strategies of clonal palms (Arecaceae) in a neotropical rainforest, Yasuní, Ecuador. Australian Journal of Botany 48:167-178. DOI: http://dx.doi.org/10.1071/ BT98048

Tomlinson P.B. 1973a. Branching in Monocotyledons. The Quarterly Review of Biology, 48(3):458-466.

Tomlinson P.B. 1973b. Establishment Growth in Woody Monocotyledons Native to New Zealand. New Zealand Journal of Botany, 11(4): 627-644.

Tomlinson P. B. 1990. The Structural Biology of Palms. New York: Oxford University Press. 490 p. 